\title{
REAL INTERPOLATION FOR DIVISIBLE CONES
}

\author{
by MARÍA J. CARRO,* STEFAN ERICSSON and LARS-ERIK PERSSON
}

(Received 21st March 1997)

We give necessary and sufficient conditions on a general cone of positive functions to satisfy the Decomposition Property (DP) introduced in [5] and connect the results with the theory of interpolation of cones introduced by Sagher [9]. One of our main result states that if $Q$ satisfies DP or equivalently is divisible, then for the quasi-normed spaces $E_{0}$ and $E_{1}$,

$$
\left(Q \cap E_{0}, Q \cap E_{1}\right)_{\theta, q}=Q \cap\left(E_{0}^{Q}, E_{1}^{Q}\right)_{\theta, q}
$$

where $E^{Q}=\{f ; \tilde{Q} f \in E\}$ with $\tilde{Q} f=\inf \{g \in Q ;|f| \leq g\}$.

According to this formula, it yields that the interpolation theory for divisible cones can be easily obtained from the classical theory.

1991 Mathematics subject classification: 46M35.

\section{Introduction and preliminaries}

Given a vector space $V$, a set $Q \subset V$ is called a cone if $Q+Q \subset Q$ and $\lambda Q \subset Q$, for every $\lambda>0$.

In [9], the author gives the definition of the interpolation spaces $\left(Q_{0}, Q_{1}\right)_{0, q}$ where $Q_{i}$ is a cone instead of a quasi-normed space $E_{i}$, as in the classical case (see [2]).

In particular, if $Q$ is a cone contained in the sum space $E_{0}+E_{1}$ and we consider $Q_{i}=Q \cap E_{i}$, it yields that

$$
\left(Q_{0}, Q_{1}\right)_{0 . q}=\left\{f \in Q ;\left(\int_{0}^{\infty}\left(\frac{K^{Q}(t, f ; \bar{E})}{t^{\theta}}\right)^{q} \frac{d t}{t}\right)^{1 / q}<\infty\right\}
$$

where

$$
K^{Q}(t, f ; \bar{E})=\inf \left\{\left\|f_{0}\right\|_{E_{0}}+t\left\|f_{1}\right\|_{E_{1}}: f=f_{0}+f_{1}, f_{i} \in Q\right\}
$$

From this fact, it follows immediately that

* This work has been partly supported by the DGICYT PB94-0879. 


$$
\left(Q_{0}, Q_{1}\right)_{\theta, q} \subset Q \cap\left(E_{0}, E_{1}\right)_{\theta, q}
$$

and a cone $Q \subset E_{0}+E_{1}$ is called a Marcinkiewicz cone (MC) (see [9]) if, for every $0<\theta<1$ and every $0<q \leq \infty$,

$$
\left(Q_{0}, Q_{1}\right)_{\theta, q}=Q \cap\left(E_{0}, E_{1}\right)_{0, q} .
$$

To get such an equality is not an easy task, but it follows immediately if we can show that, for every $f \in Q$,

$$
K^{Q}(t, f ; \bar{E}) \approx K(t, f ; \bar{E}) .
$$

For the case when $Q=D_{c}$ is the cone of positive and decreasing right-continuous functions on $(0, \infty)$, this has been studied in [5] where the authors define Marcinkiewicz pairs as those pairs $\bar{E}$ satisfying (1).

For this purpose, it turns out to be fundamental what they called the Decomposition Property for cones:

Definition (Decomposition Property). We say that a cone $Q$ satisfies the Decomposition Property or simply $Q$ satisfies DP, if the following condition holds: given $f, f_{0}, f_{1} \in Q$ so that $f \leq f_{0}+f_{1}$, there exists $\phi_{0}, \phi_{1} \in Q$ such that $\phi_{0} \leq f_{0}, \phi_{1} \leq f_{1}$ and $f=\phi_{0}+\phi_{1}$.

In [5] the Decomposition Property is proved for $Q=D_{c}$.

Another example of a cone satisfying DP is given by the cone $Q=C$ of positive concave functions in $(0, \infty)$ (see [3]). Moreover, the Decomposition Property for this cone is fundamental in the study of the $K$-divisibility property for compatible couples $([1,3])$.

The results of [9] and [5] have had interesting applications in the setting of Fourier series and in the theory of boundedness of operators acting on decreasing functions, respectively.

The paper is organized as follows. In Section 2, we give necessary and sufficient conditions on a general cone of positive functions to satisfy DP and in Section 3, we establish the connection of this property with the theory of interpolation of cones, we have mentioned above. Section 4 is devoted to present some formulae for the $\mathrm{K}$ functional for different divisible cones.

Important remark. In Section 2, we deal with a cone $Q$ of pointwise defined functions on an arbitrary set $\Omega$ and hence $f=g$ means $f(x)=g(x)$ for every $x \in \Omega$, while in Section 3, we work with a cone $[Q]$ of equivalence classes of measurable functions defined on a $\sigma$-finite measure space $\Omega$ and consequently $f=g$ means $f(x)=g(x)$ almost everywhere. (We use the same convention for the symbols $\leq$ and $\geq$ ).

Also, we shall write $f \lesssim g$ or $g \gtrsim f$ if there exists a strictly positive constant $M$ such 
that, $f \leq M g$. If $f \lesssim g$ and $g \lesssim f, f$ and $g$ are said to be equivalent and we write $f \approx g$.

Acknowledgment. The first named author wants to express her gratitude to the department of Mathematics at Lulea University for their hospitality while she was visiting Lulea in the period August-December 1996. We also thank the referee for some good advice which has improved the final version of the paper.

\section{Some general results concerning the Decomposition Property}

Let $\mathcal{F}(\Omega)$ be the set of all real valued functions defined on an arbitrary set $\Omega$ and let $\overline{\mathcal{F}}(\Omega)$ be $\mathcal{F}(\Omega)$ together with the constant function $+\infty$.

Let us consider an arbitrary nonempty cone $Q$ of $\mathcal{F}(\Omega)$. Throughout the paper the functions in $Q$ are assumed to take nonnegative values. To this cone, we associate the sublinear function $\tilde{Q}: \mathcal{F} \rightarrow \overline{\mathcal{F}}$ defined as

$$
\tilde{Q} f:=\inf \{g \in Q ;|f| \leq g\}
$$

where $\tilde{Q} f \equiv+\infty$ when no $g \in Q$ majorizes $|f|$.

Let us define, for $x, y \in \Omega$,

$$
h^{Q}(x, y):=\inf \left\{\frac{f(y)}{f(x)} ; f \in Q, f(x) \neq 0\right\} .
$$

In what follows this function will be fundamental.

We also need to deal with an explicit formula for $\tilde{Q} f$ and this will be given in the next proposition. We observe that if $\inf _{i} f_{i} \in Q$ whenever $f_{i} \in Q$ for every $i$, then one can easily see that

$$
\tilde{Q} f(y)=\inf _{h \in Q}\left[\sup _{x}\left(\frac{|f(x)|}{h(x)}\right) h(y)\right] .
$$

However, for our purpose, it will be very much useful to interchange the inf and sup in the above expression.

Proposition 2.1. Let $Q$ be a cone and let us define, for $y \in \Omega$,

$$
F_{f}^{Q}(y):=\sup _{x}\left(|f(x)| h^{Q}(x, y)\right)
$$

(i) If $F_{f}^{Q} \in Q$, then $F_{f}^{Q}=\tilde{Q} f$.

(ii) If there exists $y \in \Omega$ such that $F_{f}^{Q}(y)=+\infty$, then $\tilde{Q} f \equiv+\infty$. 
Proof. (i) First we note that

$$
F_{f}^{Q}(y) \geq|f(y)| h^{Q}(y, y)=|f(y)|,
$$

and hence $F_{f}^{Q}$ is a majorant of $|f|$ and thus, $\tilde{Q} f \leq F_{f}^{Q}$. On the other hand, if $g \in Q$ is a majorant of $|f|$, then

$$
F_{f}^{Q}(y) \leq \sup _{x}\left(g(x) \inf _{r \in Q} \frac{r(y)}{r(x)}\right) \leq \sup _{x}\left(g(x) \frac{g(y)}{g(x)}\right)=g(y),
$$

and since $F_{f}^{Q} \in Q$, it follows that $F_{f}^{Q} \leq \tilde{Q} f$ and the proof is complete.

(ii) By hypothesis, given $\lambda>0$, there exists an $x$ such that $\lambda<|f(x)| h^{Q}(x, y)$. Assume that $\tilde{Q} f$ is finite; that is, there exists $g \in Q$ which is a majorant of $|f|$. Then $\lambda<|f(x)| h^{Q}(x, y) \leq g(x) h^{Q}(x, y) \leq g(y)$. Hence $g \notin Q$ and we are done.

Let now $\Psi_{Q}$ be the collection of sets of the type $\{x \in \Omega ; f(x) \geq g(x)\}$ for $f, g \in Q$.

Then, our first main result is the following:

Theorem 2.2. Assume that the cone $Q \subset \mathcal{F}(\Omega)$ satisfies the following conditions:

(a) $F_{f}^{Q} \in Q$ for all $f \in \mathcal{F}$ such that $F_{f}^{Q}$ is finite.

(b) If $g_{0}, g_{1} \in Q$ and $E \in \Psi_{Q}$ are such that $\tilde{Q}\left(g_{i} \chi_{E}\right)=g_{i}$, for $i=0,1$, then $\tilde{Q}\left(\left(g_{0}+g_{1}\right) \chi_{E}\right)=$ $g_{0}+g_{1}$.

Then, $Q$ satisfies $D P$.

Proof. First, we observe that (a) implies that if $\left\{f_{\alpha}\right\}$ is a linearly ordered subset of $Q$, then inf $f_{\alpha} \in Q$. In fact, since $f_{\alpha} \in Q$ and (a) holds, we have that $f_{\alpha}=\sup _{x} f_{\alpha}(x) h^{Q}(x, \cdot)$ and, by taking infimum on both sides, we find that

$$
\inf _{\alpha} f_{\alpha} \leq \sup _{x}\left[\inf _{\alpha} f_{\alpha}(x) h^{Q}(x, \cdot)\right] \leq \inf _{\alpha} f_{\alpha}
$$

which again is in $Q$ by (a).

Take $f, f_{0}, f_{1}$ in $Q$ such that $f \leq f_{0}+f_{1}$. We begin by following the proof of Theorem 3.2.2 from [3]. Consider the set

$$
A:=\left\{(g, h) \in Q \times Q ; f \leq g+h, g \leq f_{0} \text { and } h \leq f_{1}\right\} .
$$

On $A$ we define the order

$$
(g, h) \leq\left(g^{\prime}, h^{\prime}\right) \Leftrightarrow g \leq g^{\prime} \text { and } h \leq h^{\prime} .
$$

We will now show that a minimal element of $A$ gives the decomposition of $f$ that we are looking for. Let $\left(g_{0}, g_{1}\right)$ be a minimal element in $A$, which exists by Zorn's Lemma 
and (a), and let us prove that $f=g_{0}+g_{1}$. Assume that, for some $\alpha_{0}<1$, and for every $\alpha \in\left[\alpha_{0}, 1\right)$, the set

$$
E_{\alpha}:=\left\{x ; f(x)<\alpha\left(g_{0}(x)+g_{1}(x)\right)\right\},
$$

is nonempty. Hence since $\tilde{Q} f=f$ and

$$
f=\frac{f g_{0}}{g_{0}+g_{1}}+\frac{f g_{1}}{g_{0}+g_{1}},
$$

we get

$$
f \leq \tilde{Q}\left(\frac{f g_{0}}{g_{0}+g_{1}}\right)+\tilde{Q}\left(\frac{f g_{1}}{g_{0}+g_{1}}\right) \leq g_{0}+g_{1} .
$$

Thus, by the minimality of $\left(g_{0}, g_{1}\right)$ we have that, for $i=0,1$,

$$
\tilde{Q}\left(\frac{f g_{i}}{g_{0}+g_{1}}\right)=g_{i}
$$

which, by Proposition 2.1, implies that

$$
g_{i}(y)=\sup _{x} \frac{f(x) g_{i}(x)}{g_{0}(x)+g_{1}(x)} h^{Q}(x, y) .
$$

We now observe that it is sufficient to take supremum over $E_{\alpha}^{c}$, since,

$$
\sup _{x \in E_{3}} \frac{f(x) g_{i}(x)}{g_{0}(x)+g_{1}(x)} h^{Q}(x, y) \leq \alpha \sup _{x \in E_{3}} g_{i}(x) h^{Q}(x, y)<g_{i}(y) .
$$

Hence,

$$
g_{i}(y)=\sup _{x \in E_{2}^{\complement}} \frac{f(x) g_{i}(x)}{g_{0}(x)+g_{1}(x)} h^{Q}(x, y) \leq \sup _{x \in E_{z}^{E}} g_{i}(x) h^{Q}(x, y) \leq g_{i}(y),
$$

and we have that $\tilde{Q}\left(g_{i} \chi_{E_{s}^{E}}\right)=g_{i}$. Hence, it follows from (b) that

$$
\begin{aligned}
\left(g_{0}+g_{1}\right) \chi_{E_{1}} & =\left(\tilde{Q}\left(g_{0} \chi_{E_{z}^{E}}\right)+\tilde{Q}\left(g_{1} \chi_{E_{z}^{E}}\right)\right) \chi_{E_{1}}=\tilde{Q}\left(\left(g_{0}+g_{1}\right) \chi_{E_{2}^{E}}\right) \chi_{E_{1}} \\
& \leq \frac{1}{\alpha} \tilde{Q}\left(f \chi_{E_{z}}\right) \chi_{E_{1}} \leq \frac{1}{\alpha} f \chi_{E_{1}} .
\end{aligned}
$$

and letting $\alpha$ go to 1 , we find that

$$
\left(g_{0}+g_{1}\right) \chi_{E_{1}} \leq f \chi_{E_{1}}<\left(g_{0}+g_{1}\right) \chi_{E_{1}},
$$


which is a contradiction. Therefore we conclude that $E_{\alpha}=\emptyset$ for every $0<\alpha<1$ and hence $f=g_{0}+g_{1}$ which finishes the proof.

The next result shows that, under a fairly mild restriction, condition (b) above is also necessary.

Theorem 2.3. If a cone $Q \subset \mathcal{F}(\Omega)$ satisfies $D P$ and for every $g \in Q$ and every set $E, \tilde{Q}\left(g \chi_{E}\right) \in Q$, then $(b)$ holds for every set $E \subset \Omega$.

Proof. Let us assume that (b) does not hold. Then there exist $g_{0}$ and $g_{1}$ in $Q$ and $E$ so that $\tilde{Q}\left(g_{i} \chi_{E}\right)=g_{i}$ and

$$
\tilde{Q}\left(\left(g_{0}+g_{1}\right) \chi_{E}\right) \neq g_{0}+g_{1} .
$$

Since the Decomposition Property holds, we have that $\tilde{Q}\left(\left(g_{0}+g_{1}\right) \chi_{E}\right)=h_{0}+h_{1}$ for some $h_{i} \leq g_{i}, h_{i} \in Q$. Obviously, $h_{i} \chi_{E}=g_{i} \chi_{E}$ and hence $\tilde{Q}\left(h_{i} \chi_{E}\right)=g_{i}$. Therefore,

$$
\tilde{Q}\left(\left(g_{0}+g_{1}\right) \chi_{E}\right)=h_{0}+h_{1} \geq \tilde{Q}\left(h_{0} \chi_{E}\right)+\tilde{Q}\left(h_{1} \chi_{E}\right)=g_{0}+g_{1} \geq \tilde{Q}\left(\left(g_{0}+g_{1}\right) \chi_{E}\right),
$$

which contradicts (2) and we are done.

In the sequel, we say that $Q$ is a divisible cone if it satisfies DP and a non-divisible cone if it does not.

\subsection{Divisible cones}

We already know that the cone $C$ of concave and the cone $D_{c}$ of decreasing right continuous functions on $\mathbb{R}^{+}$are divisible cones.

We should point out that it is very easy to check that the cone $D$ of decreasing functions on $\mathbb{R}^{+}$satisfies the conditions in Theorem 2.2 and hence, we obtain a proof of Cerdà and Martin's result [5, Lemma 1] without assuming that the functions are right continuous. On the contrary, this is not the case for $C$, since although this cone satisfies (b), it fails to satisfy condition (a).

Also, if a cone satisfies that given $f_{0}$ and $f_{1}$ in the cone, the functions $f_{0} f_{1}$ and $f_{0} / f_{1}$ belong to $Q$ (whenever it is well defined), then the Decomposition Property holds trivially. Hence we have, for example, that the cone of positive and radial functions or the cone of positive and periodic functions on $\mathbb{R}^{n}$ are in the set of divisible cones.

Let us give a list of other divisible cones:

1. Decreasing sequences in $\mathbb{Z}$.

2. Quasi-monotone sequences (see [9]); that is, those sequences $\left(a_{n}\right)_{n}$ so that $a_{n} / n^{\beta}$ is decreasing for some $\beta>0$.

3. Sequences $\left(a_{n}\right)_{n}$ so that $a_{n} / b_{n}$ is decreasing for some fixed sequence $\left(b_{n}\right)$. 
4. The cone of all functions $f$ so that $f h$ is decreasing for a fixed function $h$.

5. Increasing functions (and all the above examples with the increasing property instead of decreasing).

However, although one can easily see that all these cones satisfy the conditions (a) and (b) of our Theorem 2.2, the Decomposition Property can be proved as an easy consequence of the decreasing case $D$.

Let us consider now the following cone: let $A$ be any set in $\mathbb{R}^{+}$and let us say that a function $f$ is decreasing in $A$ if, for every $x, y \in A, x \geq y$ we have $f(x) \leq f(y)$. If

$$
D^{A}=\left\{f: \mathbb{R}^{+} \rightarrow \mathbb{R}^{+} ; f \text { is decreasing in } A\right\},
$$

then $D^{A}$ is a divisible cone.

To see this, we first observe that

$$
h^{D^{A}}(x, y)= \begin{cases}1 & \text { if } x=y \\ 1 & \text { if } x \geq y \text { and } x, y \in A \\ 0 & \text { elsewhere }\end{cases}
$$

Hence,

$$
F_{f}^{D^{A}}(y)= \begin{cases}|f(y)| & \text { if } y \notin A, \\ \sup _{x \geq y}\left|f(x) \chi_{A}(x)\right| & \text { if } y \in A,\end{cases}
$$

and condition (a) holds,

Also, for a function $f \in D^{A}$, we can deduce from the previous expression that $\widetilde{D^{A}}\left(f \chi_{E}\right)=f$ if and only if

$$
f(y)= \begin{cases}0 & \text { if } y \notin E \cup A, \\ \lim _{x \rightarrow x_{y}, x \in A \cap E} f(x) & \text { if } y \in A \text { and } x_{y} \notin A \cap E \cap[y, \infty), \\ f\left(x_{y}\right) & \text { if } y \in A \text { and } x_{y} \in A \cap E \cap[y, \infty),\end{cases}
$$

where $x_{y}=\inf A \cap E \cap[y, \infty)$ and the above limit is zero whenever $A \cap E \cap[y, \infty)=\emptyset$. From this, also condition (b) is easily checked.

Using the same technique one can easily see that, for a collection of sets $\left\{A_{i}\right\}_{i}$ in an ordered measure set $\Omega$ (say, $\mathbb{R}^{+}$or $\mathbb{Z}$ ), also

$$
Q=\left\{f: \Omega \rightarrow \mathbb{R}^{+} ; f \text { is decreasing in } A_{i}, \forall i\right\}
$$

is a divisible cone. 


\subsection{Non-divisible cones}

Using Theorem 2.3 we can also give some examples of non-divisible cones.

Example 1. Let $Q=Q C$ be the cone of quasi-concave functions; that is, increasing functions so that $f(x) / x$ is decreasing. If we consider

$$
g_{0}(y)=\left\{\begin{array}{ll}
1 & \text { if } y \leq 1, \\
y & \text { if } y>1,
\end{array} \quad g_{1}(y)= \begin{cases}1 & \text { if } y \leq 1 / 2 \\
2 y & \text { if } y>1 / 2\end{cases}\right.
$$

and $E=(0,1 / 3] \cup[8 / 3, \infty)$, then one can easily see that $\tilde{Q}\left(g_{i} \chi_{E}\right)=g_{i}(i=0,1)$ but

$$
\tilde{Q}\left(\left(g_{0}+g_{1}\right) \chi_{E}\right)= \begin{cases}2 & \text { if } y \leq 2 / 3 \\ 3 y & \text { if } y>2 / 3\end{cases}
$$

and hence $\tilde{Q}\left(\left(g_{0}+g_{1}\right) \chi_{E}\right) \neq g_{0}+g_{1}$. Therefore, $Q C$ is a non-divisible cone.

Similarly, one can easily see that, if $h_{1}$ is an increasing function, $h_{2}$ is decreasing and $h_{1} / h_{2}$ is a bijection, then

$$
Q_{h_{1}}^{h_{2}}=\left\{f: \mathbb{R}^{+} \rightarrow \mathbb{R}^{+} ; \quad f h_{1} \text { increases and } f h_{2} \text { decreases }\right\}
$$

is a non-divisible cone. To see this, we only have to observe that $f \in Q_{h_{1}}^{h_{2}}$ if and only if $g_{0}\left(f \circ g_{1}\right) \in Q C$ where

$$
g_{0}=h_{1} \circ\left(\frac{h_{1}}{h_{2}}\right)^{-1} \quad \text { and } \quad g_{1}=\left(\frac{h_{1}}{h_{2}}\right)^{-1} .
$$

Hence, we get that $Q_{h_{1}}^{h_{2}}$ is a non-divisible cone since this holds for $Q C$ and $g_{j}(j=0,1)$ are increasing functions.

In particular, given $\alpha>\beta$, the cone

$$
Q_{\alpha}^{\beta}=\left\{f: \mathbb{R}^{+} \rightarrow \mathbb{R}^{+} ; \quad f(x) x^{\alpha} \text { increases and } f(x) x^{\beta} \text { decreases }\right\}
$$

is a non-divisible cone.

Example 2. Let us now consider the cone $Q=D_{n}$ of positive functions on $\mathbb{R}_{+}^{n}$ so that they are decreasing in each variable. For simplicity, we shall restrict ourselves to the case $n=2$.

If $\bar{z}=\left(z_{1}, z_{2}\right) \in \mathbb{R}_{+}^{2}$ and by $\bar{x} \leq \bar{y}$ we mean $x_{i} \leq y_{i}, i=1,2$, we find that

$$
h^{Q}(\bar{x}, \bar{y})= \begin{cases}1 & \text { if } \bar{y} \leq \bar{x} \\ 0 & \text { elsewhere }\end{cases}
$$

and, thus, 


$$
F_{f}(\bar{y})=\sup _{\bar{x} \geq \bar{y}}|f(\bar{x})|
$$

Therefore, if we take $E=\{(1,2),(2,1)\}$, then

$$
g_{0}(x, y)= \begin{cases}3 & \text { if } 0<x \leq 1 \text { and } 0<y \leq 2 \\ 2 & \text { if } 1<x \leq 2 \text { and } 0<y \leq 1 \\ 0 & \text { elsewhere }\end{cases}
$$

and

$$
g_{1}(x, y)= \begin{cases}7 & \text { if } 0<x \leq 2 \text { and } 0<y \leq 1 \\ 3 & \text { if } 0<x \leq 1 \text { and } 1<y \leq 2 \\ 0 & \text { elsewhere }\end{cases}
$$

so that $\tilde{Q}\left(g_{i} \chi_{E}\right)=g_{i}$ but $\tilde{Q}\left(\left(g_{0}+g_{1}\right) \chi_{E}\right) \neq g_{0}+g_{1}$ and, hence, $D_{2}$ and similarly $D_{n}$ are non-divisible cones.

\section{Connection between the Decomposition Property and interpolation theory}

Let now $L_{0}(\Omega)$ be the set of all equivalence class [f] of real valued measurable functions defined on a $\sigma$-finite measure space $(\Omega, \Sigma, \mu)$.

A function lattice $E$ is understood to be a quasi-normed space in $L_{0}(\Omega)$ which satisfies that if $|f| \leq|g|$ a.e. and $g \in E$, then $f \in E$ and $\|f\| \leq\|g\|$.

Throughout, the convention that $\|f\|_{X}=+\infty$ for $f \notin X$ will be used and $X=Y$ will mean that $X$ and $Y$ are equal as sets and their quasi-norms are equivalent.

Also the letter $Q$ will indicate through the rest of the paper a cone of measurable functions pointwise defined on $\Omega$ while we shall write

$$
[Q]=\{[f] ; f \in Q\}
$$

From now on, we shall write as usual $f, g, \ldots$ instead of $[f],[g], \ldots$ when it is clear from the context but we shall try to be precise with the notation $Q$ and $[Q]$. However, when working with a couple of lattices $\bar{E}$, we shall write $K^{Q}(t, f ; \bar{E})$ to indicate $K^{(Q)}(t,[f] ; \bar{E})$.

Following the notation in the previous section, let $\bar{L}_{0}(\Omega)$ be $L_{0}(\Omega)$ together with the constant function $+\infty$ and set $\tilde{Q}: L_{0} \rightarrow \bar{L}_{0}$ defined by

$$
\tilde{Q} f:=\inf \{g \in[Q] ;|f| \leq g\}
$$

in the sense that either $\bar{Q} f \equiv \infty$ or $\tilde{Q} f$ is a measurable function so that $|f| \leq \tilde{Q} f$ and for every $g \in[Q]$ so that $|f| \leq g$ we have $\tilde{Q} f \leq g$.

For a function lattice $E$, we define

$$
E^{Q}:=\left\{f \in L_{0} ; \tilde{Q} f \in E\right\}
$$




\section{MARÍA J. CARRO, STEFAN ERICSSON AND LARS-ERIK PERSSON}

which will be a function lattice under the quasi-norm

$$
\|f\|_{E^{\ell}}:=\|\tilde{Q} f\|_{E}
$$

Clearly, we have $E^{Q} \hookrightarrow E$, and if $\tilde{Q}$ is bounded from $E$ into $E$, then $E=E^{Q}$. We will also consider $F^{Q}$, with the obvious definition, when $F$ is only a subset of a lattice. It trivially holds that

$$
[Q] \cap E \hookrightarrow E^{Q} \hookrightarrow E
$$

Throughout the rest of the paper, we shall assume that $\tilde{Q} f$ is measurable, for every $f \in L_{0}(\Omega)$ and when working with $\vec{E}^{Q}=\left(E_{0}^{Q}, E_{1}^{Q}\right)$ we shall also assume that

$$
\tilde{Q} f \in[Q] \quad \text { for all } f \in\left(E_{0}+E_{1}\right)^{Q} \text {. }
$$

Moreover,

Theorem 3.1. If $[Q]$ satisfies $D P$, then

$$
K\left(t, f ; \bar{E}^{Q}\right)=K^{Q}(t, \tilde{Q} f ; \bar{E}) .
$$

Proof. Let $\tilde{Q} f=f_{0}+f_{1}$, with $f_{i} \in[Q]$. Then, according to the lattice property, we find that

$$
K\left(t, f ; \bar{E}^{Q}\right) \leq K\left(t, \tilde{Q} f ; \bar{E}^{Q}\right) \leq\left\|f_{0}\right\|_{E_{0}}+t\left\|f_{1}\right\|_{E_{1}} .
$$

For the converse, we take $f=f_{0}+f_{1}$. According to the sublinearity property of $\tilde{Q}$, we have that $\tilde{Q} f \leq \bar{Q} f_{0}+\tilde{Q} f_{1}$ and, by the Decomposition Property, there exist $\phi_{0}, \phi_{1} \in[Q]$ such that $\tilde{Q} f=\phi_{0}+\phi_{1}$ and $\phi_{i} \leq \tilde{Q} f_{i}, i=0,1$. Hence

$$
K^{Q}(t, \tilde{Q} f ; \bar{E}) \leq\left\|\phi_{0}\right\|_{E_{0}}+t\left\|\phi_{1}\right\|_{E_{1}} \leq\left\|\tilde{Q} f_{0}\right\|_{E_{0}}+\left\|\tilde{Q} f_{1}\right\|_{E_{1}}=\left\|f_{0}\right\|_{E_{0}^{Q}}+t\left\|f_{1}\right\|_{E_{1}^{Q}}
$$

and the proof follows by taking the infimum.

In particular, we have that if $[Q]$ is divisible,

$$
K^{Q}(t, f ; \bar{E})=K\left(t, f ; \bar{E}^{Q}\right),
$$

for every $f \in[Q]$ and then, the following useful corollary is easily obtained.

Corollary 3.2. If $[Q]$ satisfies $D P$ and $Q_{i}=E_{i} \cap[Q]$, then

$$
\left(Q_{0}, Q_{1}\right)_{0, q}=[Q] \cap\left(E_{0}^{Q}, E_{1}^{Q}\right)_{\theta, q},
$$

for all $0<\theta<1$ and $0<q \leq \infty$. 
Remark 3.3. (a) Since $[Q] \cap E_{i}=[Q] \cap E_{i}^{Q}$, it follows from Corollary 3.2, that if $[Q]$ satisfies DP, then $[Q]$ is a MC with respect to the couple $\left(E_{0}^{Q}, E_{1}^{Q}\right)$, (this fact also follows from Lemma 2 in [6]).

(b) Since $\tilde{Q}: E_{i}^{Q} \rightarrow E_{i}^{Q}$ is bounded, it follows by interpolation that

$$
\left(E_{0}^{Q}, E_{1}^{Q}\right)_{0, q}=\left(E_{0}^{Q}, E_{1}^{Q}\right)_{0, q}^{Q} \text {. }
$$

(c) Following the notation in [4], we have that $Q_{i}=Q_{E_{i}, \tilde{Q}}$ and hence formula (4) could also be obtained from Remark 2.4 above and Theorem 2 in the mentioned paper. However, with this technique we can only obtain $K^{Q}(t, f ; \bar{E}) \approx K\left(t, f ; \bar{E}^{Q}\right)$ but not the equality.

(d) We should point out that the Decomposition Property for cones is connected with the $S$-divisibility property introduced in [4], in the following sense: if a cone $[Q]$ satisfies $\mathrm{DP}$, then $[Q]$ is $\tilde{Q}$-divisible with respect to $[Q]$ and every couple of lattices $\bar{E}$.

Now, using Corollary 3.2 and expression (5), we can obtain the following reiteration formula which, in the case $Q=D_{c}$, was proved in [5]:

Theorem 3.4. If $[Q]$ satisfies $D P$ and $Q_{i}=E_{i} \cap[Q]$, then

$$
\left(\left(Q_{0}, Q_{1}\right)_{o_{0, q_{0}}},\left(Q_{0}, Q_{1}\right)_{0_{1}, q_{1}}\right)_{\lambda, q}=\left(Q_{0}, Q_{1}\right)_{0, q},
$$

where $\theta_{0} \neq \theta_{1}$ and $\theta=(1-\lambda) \theta_{0}+\lambda \theta_{1}$.

Proof. By Corollary 3.2, (5) and the classical reiteration theorem, we obtain that

$$
\begin{aligned}
\left(\left(Q_{0}, Q_{1}\right)_{0_{0}, q_{0}},\left(Q_{0}, Q_{1}\right)_{0_{1}, q_{1}}\right)_{\lambda, q} & =\left([Q] \cap\left(E_{0}^{Q}, E_{1}^{Q}\right)_{0_{0, q_{0}}},[Q] \cap\left(E_{0}^{Q}, E_{1}^{Q}\right)_{0_{1, q_{1}}}\right)_{\lambda, q} \\
& =[Q] \cap\left(\left(E_{0}^{Q}, E_{1}^{Q}\right)_{0_{0, q_{0}}}^{Q},\left(E_{0}^{Q}, E_{1}^{Q}\right)_{0_{1, q}}^{Q}\right)_{\lambda, q} \\
& =[Q] \cap\left(\left(E_{0}^{Q}, E_{1}^{Q}\right)_{0_{0, q_{0}}},\left(E_{0}^{Q}, E_{1}^{Q}\right)_{0_{1}, q_{1}}\right)_{\lambda, q} \\
& =[Q] \cap\left(E_{0}^{Q}, E_{1}^{Q}\right)_{0, q}=\left(Q_{0}, Q_{1}\right)_{0, q} .
\end{aligned}
$$

Similar technique can be used to prove the following extension of the Holmstedt formula (see also [5] for the decreasing case):

Theorem 3.5. If $[Q]$ satisfies $D P$ and $Q_{i}=E_{i} \cap[Q]$, then

$$
K\left(t, f ;\left(Q_{0}, Q_{1}\right)_{0_{0, q_{0}}},\left(Q_{0}, Q_{1}\right)_{\theta_{1}, q_{1}}\right) \approx\left[\int_{0}^{t^{1 / 2}}\left(\frac{K^{Q}(t, f ; \bar{E})}{t^{O_{0}}}\right)^{q_{0}} \frac{d t}{t}\right]^{1 / q_{0}}+\left[\int_{t^{1 / 4}}^{\infty}\left(\frac{K^{Q}(t, f ; \bar{E})}{t^{O_{1}}}\right)^{q_{1}} \frac{d t}{t}\right]^{1 / q_{1}},
$$

where $0 \leq \theta_{0}<\theta_{1} \leq 1$ and $\lambda=\theta_{1}-\theta_{0}$. 
Proof. Let $f \in[Q]$. Then

$$
\begin{aligned}
K\left(t, f ;\left(Q_{0}, Q_{1}\right)_{\theta_{0}, q_{0}},\left(Q_{0}, Q_{1}\right)_{0_{1}, q_{1}}\right) & =K\left(t, f ;[Q] \cap\left(E_{0}^{Q}, E_{1}^{Q}\right)_{\theta_{0}, q_{0}},[Q] \cap\left(E_{0}^{Q}, E_{1}^{Q}\right)_{0_{1}, q_{1}}\right) \\
& =K^{Q}\left(t, f ;\left(E_{0}^{Q}, E_{1}^{Q}\right)_{\theta_{0}, q_{0}},\left(E_{0}^{Q}, E_{1}^{Q}\right)_{\theta_{1}, q_{1}}\right) \\
& =K\left(t, f ;\left(E_{0}^{Q}, E_{1}^{Q}\right)_{\theta_{0}, q_{0}}^{Q},\left(E_{0}^{Q}, E_{1}^{Q}\right)_{\theta_{1}, q_{1}}^{Q}\right) \\
& =K\left(t, f ;\left(E_{0}^{Q}, E_{1}^{Q}\right)_{\theta_{0}, q_{0}},\left(E_{0}^{Q}, E_{1}^{Q}\right)_{\theta_{1}, q_{1}}\right)
\end{aligned}
$$

and the result now follows from the classical Holmstedt formula and (4).

Remark 3.6. Let $\bar{F}=\left(F_{0}, F_{1}\right)$ be a compatible couple of lattices. Using again (4) and the general reiteration formula (see $[1,8,4]$ ) we get that if

$$
\bar{E}_{F ; K^{Q}}:=\left\{f \in\left(E_{0}+E_{1}\right) \cap[Q] ; K^{Q}(\cdot, f ; \bar{E}) \in F\right\},
$$

then

$$
K\left(t, f ; \bar{E}_{F_{0} ; K^{Q}}, \bar{E}_{F_{1} ; K^{Q}}\right) \approx K\left(t, K^{Q}(\cdot, f ; \bar{E}) ; F_{0}^{C}, F_{1}^{c}\right),
$$

and, consequently, for every lattice $G$,

$$
\left(\left(E_{0}, E_{1}\right)_{F_{0} ; K^{Q}},\left(E_{0}, E_{1}\right)_{F_{1} ; K^{Q}}\right)_{G ; K}=\left(E_{0}, E_{1}\right)_{\left(F_{0}^{c}, F_{1}^{c}\right)_{G ; K^{k}}} .
$$

Moreover, if we deal with Marcinkiewicz cones (see [9]), we have the following result:

Corollary 3.7. If $[Q] \subset E_{0}+E_{1}$ is a Marcinkiewicz cone satisfying $D P$, then

$$
\left(E_{0}^{Q}, E_{1}^{Q}\right)_{\theta, q}=\left(E_{0}, E_{1}\right)_{\theta, q}^{Q}
$$

for all $0<\theta<1$ and $0<q \leq \infty$.

Proof. By Theorem 3.1, we have that, if $Q_{i}=E_{i} \cap[Q]$, then

$$
\left(E_{0}^{Q}, E_{1}^{Q}\right)_{0, q}=\left\{f \in E_{0}^{Q}+E_{1}^{Q} ; \tilde{Q} f \in\left(Q_{0}, Q_{1}\right)_{0, q}\right\}
$$

and, hence,

$$
\begin{aligned}
\left(E_{0}^{Q}, E_{1}^{Q}\right)_{\theta, q} & =\left\{f \in E_{0}^{Q}+E_{1}^{Q} ; \tilde{Q} f \in\left[\left(E_{0}, E_{1}\right)_{\theta, q} \cap[Q]\right]\right\} \\
& =\left\{f \in E_{0}^{Q}+E_{1}^{Q} ; \tilde{Q} f \in\left(E_{0}, E_{1}\right)_{\theta, q}\right\}=\left(E_{0}, E_{1}\right)_{\theta, q}^{Q}
\end{aligned}
$$

Our next step is to extend (6) to cones which are not divisible. For this purpose we have to introduce the notion of equivalent cones. 
Definition 3.8. We say that the cones $Q_{0}$ and $Q_{1}$ are c-equivalent, and we write $Q_{0} \approx Q_{1}$, if the following condition is satisfied: for every $f \in Q_{0}$ or $f \in Q_{1}$, there exists $g \in Q_{1}$ or $g \in Q_{0}$, respectively, so that $\frac{1}{c} g \leq f \leq c g$ and if so, we write $f \stackrel{\tilde{\approx}}{\approx}$.

The next result establishes the connection between two cones $Q_{i}$ which are equivalent and the corresponding equivalence for the operators $\tilde{Q}_{i}$.

Proposition 3.9. Let $c>0$ be fixed.

(a) If $Q_{0} \approx Q_{1}$, then $\tilde{Q}_{0} \stackrel{c^{2}}{\approx} \tilde{Q}_{1}$.

(b) Assume that $\tilde{Q}_{i} f \in Q_{i}$, for every $f \in Q_{1-i}(i=0,1)$. If $\tilde{Q}_{0} \approx \tilde{Q}_{1}$, then $Q_{0} \approx Q_{1}$.

Proof. (a) Let $f: \Omega \rightarrow \mathbb{R}^{+}$and let $g \in Q_{0}$ so that $f \leq g$. Let $h \in Q_{1}$ so that $f \leq g \leq h \leq c^{2} g$. Then,

$$
\bar{Q}_{1} f \leq h \leq c^{2} g
$$

and therefore $\tilde{Q}_{1} f \leq c^{2} \tilde{Q}_{0} f$.

(b) Let $f \in Q_{0}$. Since $\tilde{Q}_{0} \approx \tilde{Q}_{1}$, we find that $\frac{1}{c} \tilde{Q}_{1} f \leq \tilde{Q}_{0} f=f \leq c \tilde{Q}_{1} f$ and since $\tilde{Q}_{1} f \in Q_{1}$ there exists an equivalent function to $f$ in $Q_{1}$. Using the same argument for $f \in Q_{1}$, we get the result.

We say that a cone is quasi-divisible if it is equivalent to a divisible cone.

It is known that $C \underset{2}{\approx} Q C$ (see [3]) and, from this, we obtain that, for every $h_{1}$ and $h_{2}$ as before, the cone

$$
C_{h_{1}}^{h_{2}}=\left\{f: \mathbb{R}^{+} \rightarrow \mathbb{R}^{+} ; g_{0}\left(f \circ g_{1}\right) \in C\right\},
$$

with $g_{j}$ as in (3), is equivalent to the cone $Q_{h_{1}}^{h_{2}}$. Moreover, using that $C$ is a divisible cone, one can easily see that same holds for $C_{h_{1}}^{h_{2}}$ and, therefore, $Q_{h_{1}}^{h_{2}}$ is a quasidivisible cone.

Observe that if $Q_{0}$ and $Q_{1}$ are two cones in $\mathcal{F}(\Omega)$ so that $Q_{0} \approx Q_{1}$, then $\left[Q_{0}\right] \approx\left[Q_{1}\right]$ and hence $\left[C_{h_{1}}^{h_{2}}\right] \approx\left[Q_{h_{1}}^{h_{2}}\right]$.

Remark 3.10. (1) Obviously, if $\tilde{Q}_{0} \approx \tilde{Q}_{1}$, then $E^{Q_{0}}=E^{Q_{1}}$ and, hence, Corollary 3.7 can be extended, in the obvious way, to quasi-divisible cones.

(2) Also, it is important to observe that, for $Q_{h_{1}}^{h_{1}}$, formula (6) is a reiteration result in the following sense: We have that

$$
h^{Q_{h_{1}}^{2}}(x, y)=\min \left(\frac{h_{1}(x)}{h_{1}(y)}, \frac{h_{2}(x)}{h_{2}(y)}\right)
$$

and 


$$
\begin{aligned}
\left(\tilde{Q}_{h_{1}}^{h_{2}} f\right)(y) & =\operatorname{ess} \sup _{x}\left(|f(x)| h^{Q_{h_{1}}^{h_{2}}}(x, y)\right) \\
& =\frac{1}{h_{1}(y)} \operatorname{ess} \sup _{x}\left[|f(x)| \min \left(h_{1}(x), \frac{h_{1}(y)}{h_{2}(y)} h_{2}(x)\right)\right] \\
& \approx \frac{1}{h_{1}(y)} K\left(\frac{h_{1}(y)}{h_{2}(y)}, f ; L_{\infty}\left(h_{1}\right), L_{\infty}\left(h_{2}\right)\right)
\end{aligned}
$$

Hence, if, for a lattice $E$, we define $\hat{E}$ by $\|f\|_{\hat{E}}=\left\|\left(f \circ\left(h_{1} / h_{2}\right)\right) / h_{1}\right\|_{E}$, with $g_{j}$ as in (3), then, for $Q=Q_{h_{1}}^{h_{2}}$,

$$
\|f\|_{E^{Q}}=\|\tilde{Q} f\|_{E}=\|f\|_{\left(L_{\infty}\left(h_{1}\right), L_{\infty}\left(h_{2}\right)\right)_{E: K}}
$$

and hence $E^{Q}=\left(L_{\infty}\left(h_{1}\right), L_{\infty}\left(h_{2}\right)\right)_{\hat{E} ; K}$. Therefore, if $\bar{A}=\left(L_{\infty}\left(h_{1}\right), L_{\infty}\left(h_{2}\right)\right)$, formula (6) reads

$$
\left(\bar{A}_{\hat{E}_{0}: K}, \bar{A}_{\hat{E}_{1} ; K}\right)_{0, q}=\bar{A}_{\left(E_{0}, E_{1}\right)_{\theta, q} ; K}
$$

which also follows from the more general reiteration formula for $K$-spaces in [8] and the fact that $\left(E_{0}, E_{1}\right)_{0, q}=\left(\hat{E}_{0}, \hat{E}_{1}\right)_{0, q}$.

\section{The $K$-functional for divisible cones}

The purpose of this section is to compute some $K$-functionals for several divisible cones and for the couples $\left(L^{p_{0}}\left(w_{0}\right), L^{p_{1}}\left(w_{1}\right)\right)$ and $\left(X, L^{\infty}\right)$ where $X$ is a lattice. We shall use formula (4) from Section 3 and the fact that the $K$-functional for the couple $\bar{E}^{Q}$, can sometimes be explicitly computed as follows (see [4]):

Theorem 4.1. Let $\bar{E}$ be a compatible couple of lattices and let us assume that the function $h^{Q}(x, \cdot)$ is measurable for a.e. $x$ and that the functions $g_{j}(x)=\left\|h^{Q}(x, \cdot)\right\|_{E_{j}^{Q}}$ are also measurable. If, for each $t>0$, there exist measurable sets $B_{0}(t)$ and $B_{1}(t)$ such that $B_{0}(t) \cup B_{1}(t)=\Omega$ and

$$
\sup _{t>0} \max \left(\frac{1}{t}\left\|\chi_{B_{0}(t)} / g_{1}\right\|_{E_{0}^{Q}}, t\left\|\chi_{B_{1}(t)} / g_{0}\right\|_{E_{1}^{Q}}\right)<\infty
$$

then

$$
K\left(t, f ; \bar{E}^{Q}\right) \approx\left\|f \chi_{B_{0}(t)}\right\|_{E_{0}^{Q}}+t\left\|f \chi_{B_{1}(t)}\right\|_{E_{1}^{Q}}
$$

for all $f \in[Q]$.

Lemma 4.2. (a) The cone $[D]$ is divisible.

(b) Let $h_{1}$ and $h_{2}$ be as in Section 2. Then $\left[C_{h_{1}}^{h_{2}}\right]$ is a divisible cone. 
Proof. (a) Let $f, f_{0}, f_{1} \in[D]$ be such that $f \leq f_{0}+f_{1}$. Let $E$ be so that $|E|=0$ and

$$
\left(f \chi_{E^{c}}\right)(x) \leq\left(f_{0} \chi_{E^{c}}\right)(x)+\left(f_{1} \chi_{E^{c}}\right)(x),
$$

for every $x$. Then, $\left(f \chi_{E^{c}}\right),\left(f_{0} \chi_{E^{c}}\right)$ and $\left(f_{1} \chi_{E^{E}}\right)$ belong to the cone $D^{E^{c}}=\{g ; g$ is decreasing in $E^{c}$ \}. Since, we have shown in Section 2 that this cone is divisible, we get that there exists $g_{i} \in D^{E^{c}}(i=0,1)$ so that $\left(f \chi_{E^{c}}\right)=g_{0}+g_{1}$ and $g_{i} \leq\left(f_{i} \chi_{E^{E}}\right)$. Then $[f]=\left[g_{0}\right]+\left[g_{1}\right]$ with $\left[g_{i}\right] \leq f_{i}$. Since clearly $\left[g_{i}\right] \in[D]$, we are done. (An alternative proof can be given using the fact that the cone $D_{c}$ is divisible and if $f \in[D]$ there exists a representative in the class that it is in $D_{c}$.)

(b) Let now $f, f_{0}, f_{1} \in\left[C_{h_{1}}^{h_{2}}\right]$ be such that $f \leq f_{0}+f_{1}$ and set $E$ as in (a). Then,

$$
g_{0}(x)\left(f \circ g_{1}\right)(x) \leq g_{0}(x)\left(f_{0} \circ g_{1}\right)(x)+g_{0}(x)\left(f_{1} \circ g_{1}\right)(x),
$$

for every $x \in E^{c}$, where $g_{i}$ are as in (3). Now, since the above functions are continuous (they are concave), we get that the above inequality holds for every $x \in \Omega$ and hence, using that $C$ is a divisible cone we get that there exist $F_{0}$ and $F_{1}$ so that $g_{0}(x)\left(f \circ g_{1}\right)(x)=F_{0}(x)+F_{1}(x)$ and $F_{i}(x) \leq g_{0}(x)\left(f_{i} \circ g_{1}\right)(x)$ for every $x \in \Omega$. From this the results follows immediately.

Let us now consider the couple $\bar{E}=\left(L^{p_{0}}\left(w_{0}\right), L^{p_{1}}\left(w_{1}\right)\right)$ and let us write $W_{i}(t)=\int_{0}^{i} w_{i}(s) d s$.

Corollary 4.3. (see [4]) If $w_{0}$ and $w_{1}$ satisfy that, for every $t>0$, there exists $a_{t} \in[0, \infty]$ such that

$$
\left(\int_{0}^{a_{t}} \frac{w_{0}(s)}{W_{1}(s)^{p_{0} / p_{1}}} d s\right)^{\frac{1}{s}} \lesssim t
$$

and

$$
\left(\frac{W_{1}\left(a_{t}\right)}{W_{0}\left(a_{t}\right)^{p_{1} / p_{0}}}+\int_{a_{i}}^{\infty} \frac{w_{1}(s)}{W_{0}(s)^{p_{1} / p_{0}}} d s\right)^{\frac{1}{n}} \lesssim \frac{1}{t}
$$

then, for every decreasing function $f$,

$$
K^{D}\left(t, f ; L^{p_{0}}\left(w_{0}\right), L^{p_{1}}\left(w_{1}\right)\right) \approx\left(\int_{0}^{a_{1}} f(s)^{p_{0}} w_{0}(s) d s\right)^{\frac{1}{n}}+t\left(\int_{a_{1}}^{\infty} f(s)^{p_{1}} w_{1}(s) d s\right)^{\frac{1}{n}}
$$

In particular, the conditions (9) and (10) are satisfied in the following cases:

(i) If $w_{i}(x) \leq W_{i}(x) / x, i=0,1$, for almost every $x$ and $W_{0}(t)^{1 / p_{0}} W_{1}(t)^{-1 / p_{i}} t^{-c}$ is increasing for some $c>0$.

(ii) If there exist $\alpha>0$ and $\beta>0$ such that $\alpha p_{0}<\beta p_{1}$ and $W_{0}^{\alpha} / W_{1}^{\beta}$ increases. 
As a corollary of the previous result, one can easily get conditions on the weights $w_{0}$ and $w_{1}$ so that $K^{Q_{h}}$ can be computed for the cone $Q_{h}$ of positive functions $f$ such that $f h$ is decreasing.

If we now want to deal with the cone $Q=C_{h_{1}}^{h_{2}}$, we first need to observe that $h^{Q}=h^{Q_{m}^{n}}$ and

$$
\begin{aligned}
g_{j}(x) & =\left\|h^{Q}(x, \cdot)\right\|_{\left(L^{\prime}\left(w_{j}\right)\right)^{Q}}=\left\|h^{Q}(x, \cdot)\right\|_{L^{\prime \prime}\left(w_{j}\right)} \\
& =\left[\int_{0}^{x}\left(\frac{h_{2}(x)}{h_{2}(y)}\right)^{p^{\prime}} w_{j}(y) d y+\int_{x}^{\infty}\left(\frac{h_{1}(x)}{h_{1}(y)}\right)^{p_{j}} w_{j}(y) d y\right]^{1 / p_{1}} .
\end{aligned}
$$

Using this fact together with Theorem 4.1, we can obtain the following result:

Proposition 4.4. If $w_{0}$ and $w_{1}$ satisfy that

$$
\int_{x}^{\infty}\left(\frac{h_{1}(x)}{h_{1}(y)}\right)^{p_{0}} w_{0}(y) d y \lesssim \int_{0}^{x}\left(\frac{h_{2}(x)}{h_{2}(y)}\right)^{p_{0}} w_{0}(y) d y,
$$

or

$$
\int_{0}^{x}\left(\frac{h_{2}(x)}{h_{2}(y)}\right)^{p_{1}} w_{1}(y) d y \lesssim \int_{x}^{\infty}\left(\frac{h_{1}(x)}{h_{1}(y)}\right)^{p_{1}} w_{1}(y) d y,
$$

and, for every $t>0$, there exists $a_{t} \in[0, \infty]$ such that

$$
\left(\int_{0}^{a_{t}} \frac{w_{0}(s)}{g_{1}(s)^{p_{0}}} d s\right)^{\frac{1}{p_{0}}}+\frac{h_{1}\left(a_{t}\right)}{g_{1}\left(a_{t}\right)}\left(\int_{a_{1}}^{\infty} \frac{w_{0}(s)}{h_{1}(s)^{p_{0}}} d s\right)^{\frac{1}{\omega}} \lesssim t
$$

and

$$
\frac{h_{2}\left(a_{t}\right)}{g_{0}\left(a_{t}\right)}\left(\int_{0}^{a_{t}} \frac{w_{1}(s)}{h_{2}(s)^{p_{1}}} d s\right)^{\frac{1}{n_{1}}}+\left(\int_{a_{t}}^{\infty} \frac{w_{1}(s)}{g_{0}(s)^{p_{1}}} d s\right)^{\frac{1}{n}} \lesssim \frac{1}{t},
$$

then, for every $f \in C_{h_{1}}^{h_{2}}$,

$$
K^{c_{h_{1}}}\left(t, f ; L^{p_{0}}\left(w_{0}\right), L^{p_{1}}\left(w_{1}\right)\right) \approx\left(\int_{0}^{a_{t}} f(s)^{p_{0}} w_{0}(s) d s\right)^{\frac{1}{\omega_{1}}}+t\left(\int_{a_{1}}^{\infty} f(s)^{p_{1}} w_{1}(s) d s\right)^{\frac{1}{11}} .
$$

Proof. Let us assume that (11) holds. The proof follows similarly if instead (12) holds.

Conditions (13) and (14) imply that (7) holds and, thus, it only remains to prove that, for $f \in C_{h_{1}}^{h_{2}}$, 
$\left\|f \chi_{\left(0, a_{1}\right)}\right\|_{\left(L^{\infty}\left(w_{0}\right)\right)^{e}}+t\left\|f \chi_{(a, \infty)}\right\|_{\left(L^{\prime \prime}\left(w_{1}\right)\right)^{Q}} \approx\left\|f \chi_{\left(0, a_{1}\right)}\right\|_{L^{\infty}\left(w_{0}\right)}+t\left\|f \chi_{(a, \infty)}\right\|_{L^{\prime \prime}\left(w_{1}\right)}$.

In fact,

$$
\left\|f \chi_{\left(0, a_{1}\right)}\right\|_{\left(L^{\infty}\left(w_{0}\right)\right)^{Q}} \approx\left\|f \chi_{\left(0, a_{t}\right)}\right\|_{L^{\infty}\left(w_{0}\right)}+\left\|f\left(a_{t}\right) \chi_{\left(a_{1}, \infty\right)} \frac{h_{1}\left(a_{t}\right)}{h_{1}}\right\|_{L^{\infty}\left(w_{0}\right)}=I+I I,
$$

and to estimate II, we have to use (11) and (13) as follows:

$$
\begin{aligned}
I I & =f\left(a_{t}\right) h_{1}\left(a_{t}\right)\left(\int_{a_{t}}^{\infty} \frac{w_{0}}{h_{1}^{p_{0}}}\right)^{1 / p_{0}} \lesssim f\left(a_{t}\right) g_{1}\left(a_{t}\right) t \\
& \approx t\left(f\left(a_{t}\right) h_{1}\left(a_{t}\right)\left(\int_{a_{t}}^{\infty} \frac{w_{1}}{h_{1}^{p_{1}}}\right)^{1 / p_{1}}+f\left(a_{t}\right) h_{2}\left(a_{t}\right)\left(\int_{0}^{a_{t}} \frac{w_{1}}{h_{2}^{p_{1}}}\right)^{1 / p_{1}}\right) \\
& \lesssim t\left(\int_{a_{t}}^{\infty} f^{p_{1}} w_{1}\right)^{1 / p_{1}}+f\left(a_{t}\right) g_{0}\left(a_{t}\right)=t\left\|f \chi_{\left(a_{t}, \infty\right)}\right\|_{L^{p^{\prime}}\left(w_{1}\right)}+f\left(a_{t}\right) g_{0}\left(a_{t}\right) \\
& =I I I+I V .
\end{aligned}
$$

Now, to estimate IV, we observe that (11) is equivalent to saying that

$$
g_{0}(x) \approx \int_{0}^{x}\left(\frac{h_{2}(x)}{h_{2}(y)}\right)^{p_{0}} w_{0}(y) d y
$$

and, hence,

$$
I V \approx f\left(a_{t}\right) h_{2}\left(a_{t}\right)\left(\int_{0}^{a_{t}} \frac{w_{0}}{h_{2}^{p_{0}}}\right)^{1 / p_{0}} \leq\left\|f \chi_{\left(0, a_{1}\right)}\right\|_{L^{N^{0}\left(w_{0}\right)}}
$$

Similarly,

$$
\begin{aligned}
t\left\|f \chi_{\left(a_{t}, \infty\right)}\right\|_{\left(L^{n}\left(w_{1}\right)\right)^{e}} & \approx t\left\|\chi_{\left(0, a_{t}\right)} f\left(a_{\imath}\right) \frac{h_{2}\left(a_{t}\right)}{h_{2}}\right\|_{L^{n}\left(w_{1}\right)}+t\left\|f \chi_{\left(a_{1}, \infty\right)}\right\|_{L^{n}\left(w_{1}\right)} \\
& =V+V .
\end{aligned}
$$

Moreover, we use (14) and the above observation on $g_{0}$, to obtain the following estimate of $\mathrm{V}$ :

$$
\begin{aligned}
V & =t f\left(a_{t}\right) h_{2}\left(a_{t}\right)\left(\int_{0}^{a_{t}} \frac{w_{1}}{h_{2}^{p_{1}}}\right)^{1 / p_{1}} \lesssim f\left(a_{t}\right) g_{0}\left(a_{t}\right) \\
& \approx f\left(a_{t}\right) h_{2}\left(a_{t}\right)\left(\int_{0}^{a_{1}} \frac{w_{0}}{h_{2}^{p_{0}}}\right)^{1 / p_{0}} \lesssim\left(\int_{0}^{a_{t}} f^{p_{0}} w_{0}\right)^{1 / p_{0}}=\left\|f \chi_{\left(0, a_{1}\right)}\right\|_{L^{\infty}\left(w_{0}\right)},
\end{aligned}
$$

from which the result follows easily. 
Our next aim is to present a concrete example of how Proposition 4.4 can be applied and, for this purpose, let us assume that the following " $B_{p}$ type" conditions hold, for $i=0,1$ :

$$
h_{1}(x)^{p_{1}} \int_{x}^{\infty} \frac{w_{i}(y)}{h_{1}(y)^{p_{1}}} d y \lesssim h_{2}(x)^{p_{1}} \int_{0}^{x} \frac{w_{i}(y)}{h_{2}(y)^{p_{1}}} d y .
$$

Let us write $U_{i}(x)=\int_{0}^{x} \frac{w_{i}(y)}{h_{h}(y)^{4}} d y$. Then, one can easily see that $g_{i}(x) \approx h_{2}(x) U_{i}(x)^{1 / p_{i}}$ and the following results follow:

Corollary 4.5. Assume that (16) holds.

(a) Let $U_{1}(\infty)=\infty$ and there exist $\alpha>0$ and $\beta>0$ so that $U_{0}^{\alpha} U_{1}^{-\beta}$ increases and $\beta p_{1}>\alpha p_{0}$, then (15) holds with $a_{t}$ so that $t=U_{0}^{1 / p_{0}}\left(a_{t}\right) U_{1}^{-1 / p_{1}}\left(a_{t}\right)$.

(b) If

$$
\frac{w_{i}(y)}{h_{2}^{p_{1}}(y)} \lesssim \frac{U_{i}(y)}{y},
$$

and there exists $c>0$ so that $U_{0}(t)^{1 / p_{0}} U_{1}(t)^{-1 / p_{1}} t^{-c}$ is increasing, then (15) holds for $a_{t}$ so that $t=U_{0}^{1 / p_{0}}\left(a_{t}\right) U_{1}^{-1 / p_{1}}\left(a_{t}\right)$.

Proof. Some straightforward calculations show that conditions (13) and (14) are satisfied.

Remark 4.6. If $h_{1}(x)=x^{\alpha}$ and $h_{2}(x)=x^{-\beta}$, then the condition (16) reads $w_{i}(x) x^{\beta p_{t}} \in$ $B_{(\alpha+f) p_{i}}$ which in the concave case reduces to $w_{i}(x) x^{p_{1}} \in B_{p_{i}}$.

Concerning the couple $\left(X, L^{\infty}\right)$ where $X$ is a lattice, we have the following result:

Theorem 4.7. Let $[Q]$ be a divisible cone such that

(i) For every $f \in[Q], \min (f, 1) \in[Q]$,

(ii) For every $f \in[Q]$, there exists $g \in[Q]$ so that $(f-1)_{+} \leq g$ and $\|g\|_{X} \lesssim$ $\left\|(f-1)_{+}\right\|_{x}$.

Then, $[Q]$ is a Marcinkiewicz cone with respect the couple $\left(X, L^{\infty}\right)$.

Proof. It is known (see [7]) that

$$
K\left(t, f ; X, L^{\infty}\right)=\inf _{\lambda>0}\left\{\left\|(f-\lambda)_{+}\right\|_{X}+t\|\min (f, \lambda)\|_{\infty}\right\}
$$

Let $f \in[Q]$. Since, $f=(f-\lambda)_{+}+\min (f, \lambda)$, we have that $f \leq g+\min (f, \lambda)$ and by (i) and the Decomposition Property of $[Q]$, we have that there exist $h_{j} \in[Q]$ so that 
$f=h_{0}+h_{1}, h_{0} \leq g$ and $h_{1} \leq \min (f, \lambda)$. Hence,

$$
\begin{aligned}
K^{Q}\left(t, f ; X, L^{\infty}\right) & \leq\left\|h_{0}\right\|_{X}+t\left\|h_{1}\right\|_{\infty} \leq\|g\|_{X}+t\|\min (f, \lambda)\|_{\infty} \\
& \lesssim\left\|(f-\lambda)_{+}\right\|_{X}+t\|\min (f, \lambda)\|_{\infty},
\end{aligned}
$$

and since $\lambda$ is arbitrary, we find that, for every $f \in[Q]$,

$$
K^{Q}\left(t, f ; X, L^{\infty}\right) \lesssim K\left(t, f ; X, L^{\infty}\right) \leq K^{Q}\left(t, f ; X, L^{\infty}\right),
$$

and the proof is complete.

Corollary 4.8. (a) $[D]$ is a Marcinkiewicz cone with respect to the couple $\left(X, L^{\infty}\right)$.

(b) If $X$ satisfies that the left translation operator $\tau_{a}: X \rightarrow X$ is uniformly bounded in $a$, then $[C]$ is a Marcinkiewicz cone with respect to the couple $\left(X, L^{\infty}\right)$.

Proof. (a) The cone $[D]$ trivially satisfies (i) and (ii) with $g=(f-1)_{+}$. Since by Lemma $4.2[D]$ is divisible we are done.

(b) Since $f$ is increasing, we can take $g=\tau_{a}\left[(f-1)_{+}\right]$for some $a$ and, hence, the conditions (i) and (ii) are satisfied.

A different proof of (a) was given in [5].

\section{REFERENCES}

1. I. U. Asekritova, The Holmstedt formula and an equivalence theorem for $\mathrm{N}$-set of Banach spaces, Yaroslav Gos. Univ. 165 (1980), 15-18.

2. J. BERGH and J. LöFSTRÖM, Interpolation Spaces. An Introduction (Grundlehren der Mathematischen Wissenschaften 223, Springer Verlag, Berlin-Heidelberg-New York, 1976).

3. YU. A. BrudNyi and N. YA. KRUGLJAK, Interpolation Functors and Interpolation Spaces (North Holland, 1991).

4. M. J. Carro, S. Ericsson and L. E. Persson, S-divisibility property and a Holmstedt type formula, J. Approx. Th., to appear.

5. J. Cerda and J. MARTIN, Interpolation of operators on decreasing functions, Math. Scand. 78 (1996), 233-245.

6. J. Cerdà and J. Martin, Interpolation restricted to decreasing functions and Lorentz spaces, Preprint (1996).

7. S. Ericsson, Exact descriptions of some $K$ and $E$ functionals, J. Approx. Th. 90 (1997), 75-87.

8. P. Nilsson, Reiteration theorems for real interpolation and approximation spaces, Ann. Math. Pura Appl. 132 (1982), 291-330. 


\section{MARÍA J. CARRO, STEFAN ERICSSON AND LARS-ERIK PERSSON}

9. Y. SAGHER, An application of interpolation theory to Fourier series, Studia Math. (1972), $169-181$.

Carro

Departament de Matemática Aplicada I ANÀLISI

UNIVERSITAT DE BARCELONA

08071 BARCELONA

SPAIN

E-mail address: carro@cerber.mat.ub.es
ERICSSON AND PERSSON

Department of Mathematics

LULE\& UNIVERSITY

S-971 87 LULEA

SWEDEN

E-mail addresses: larserik@sm.luth.se, sen@sm.luth.se 